\title{
Article \\ The Proof of a Conjecture on the Density of Sets Related to Divisibility Properties of $z(n)$
}

\author{
Eva Trojovská *(D) and Venkatachalam Kandasamy
}

Department of Mathematics, Faculty of Science, University of Hradec Králové, 50003 Hradec Králové, Czech Republic; venkatachalam.kandasamy@uhk.cz

* Correspondence: eva.trojovska@uhk.cz; Tel.: +42-049-333-2859

check for updates

Citation: Trojovská, E.; Kandasamy, V. The Proof of a Conjecture on the Density of Sets Related to Divisibility Properties of $z(n)$. Mathematics 2021, 9, 2912. https://doi.org/10.3390/ math9222912

Academic Editor: Beny Neta

Received: 21 October 2021

Accepted: 13 November 2021

Published: 16 November 2021

Publisher's Note: MDPI stays neutral with regard to jurisdictional claims in published maps and institutional affiliations.

\begin{abstract}
Let $\left(F_{n}\right)_{n}$ be the sequence of Fibonacci numbers. The order of appearance (in the Fibonacci sequence) of a positive integer $n$ is defined as $z(n)=\min \left\{k \geq 1: n \mid F_{k}\right\}$. Very recently, Trojovská and Venkatachalam proved that, for any $k \geq 1$, the number $z(n)$ is divisible by $2^{k}$, for almost all integers $n \geq 1$ (in the sense of natural density). Moreover, they posed a conjecture that implies that the same is true upon replacing $2^{k}$ by any integer $m \geq 1$. In this paper, in particular, we prove this conjecture.
\end{abstract}

Keywords: order of appearance; fibonacci numbers; divisibility; natural density; prime numbers

\section{Introduction}

Let $\left(F_{n}\right)_{n}$ be the sequence of Fibonacci numbers that is defined by the second-order recurrence

$$
F_{n+2}=F_{n+1}+F_{n},
$$

with $F_{i}=i$, for $i \in\{0,1\}$. A standard application of the Pigeonhole principle yields that for any $n \geq 1$, there exist infinitely many positive integers $m$ such that $n$ divides $F_{m}$. Thus, the arithmetic function $z(n):=\min \left\{k \geq 1: n \mid F_{k}\right\}$ is well-defined (see also ([1], p. 300) and [2] for the sharpest upper bound for $z(n)$, namely, $z(n) \leq 2 n)$. This function is known as the order of appearance in the Fibonacci sequence. The first 20 values of $z(n)$ are (see sequence A001177 in OEIS [3]):

$$
1,3,4,6,5,12,8,6,12,15,10,12,7,24,20,12,9,12,18,30 \text {. }
$$

We remark that the natural density of a set $\mathcal{A} \subseteq \mathbb{Z}_{\geq 1}$ is defined as the following limit (if it exists):

$$
\delta(\mathcal{A}):=\lim _{x \rightarrow \infty} \frac{\# \mathcal{A}(x)}{x},
$$

where $\mathcal{A}(x):=\mathcal{A} \cap[1, x]$ for $x \geq 1$ and $\# A$ denotes the cardinality of the set $A \subset \mathbb{R}$. Recently, Trojovský [4] proved that $\{n \geq 1: z(n)<\epsilon n\}$ is a set with natural density that equals to 1 , for all $\epsilon>0$, previously fixed (this generalizes the fact that $\liminf _{n \rightarrow \infty} z(n) / n$, see [5]).

Here, we are interested in some Diophantine properties of $z(n)$ (this topic has been dealt with by many authors; see, for instance, [6-12]). For any integer $m \geq 2$, we denote $\mathcal{E}_{z}^{(m)}$ as the set of all $n \in \mathbb{Z}_{\geq 1}$ for which $m$ divides $z(n)$, that is,

$$
\mathcal{E}_{z}^{(m)}:=\{n \geq 1: z(n) \equiv 0 \quad(\bmod m)\} .
$$

Recently, Trojovský ([13], Theorem 2) proved that the natural density of $\mathcal{E}_{z}^{(2)}$ is equal to 1. Moreover, Trojovská and Venkatachalam [14] generalized the previous result by showing that $\delta\left(\mathcal{E}_{z}^{\left(2^{k}\right)}\right)=1$, for all $k \geq 1$. They also posed the following conjecture: 
Conjecture 1. For all positive integers $m$, there exist positive constants $A_{m}$ and $\beta_{m}<1$ such that:

$$
\# \mathcal{E}_{z}^{(m)}(x) \geq x-A_{m} \frac{x}{(\log x)^{\beta_{m}}}
$$

for all sufficiently large $x$. In particular, $\delta\left(\mathcal{E}_{z}^{(m)}\right)=1$, for all $m \geq 1$.

In this paper, we confirm the Trojovská and Venkatachalam conjecture. For the sake of preciseness, we state it as a theorem.

Theorem 1. Conjecture 1 is true.

Although $z(n) \leq 2 n$ is the sharpest upper bound for the $z$-function (indeed, the equality holds whenever $n=6 \cdot 5^{k}, k \geq 0$ ), Marques [5], in 2013, showed that this bound can be substantially improved depending on the number of distinct prime factors of $n$. By using Marques' result, in 2020, Trojovský [15] proved that

$$
\sum_{n \leq x} \frac{z(n)}{n} \gg \log ^{2} x
$$

as $x \rightarrow \infty$.

Our next result refines the previous estimate by providing the best lower bound for the average growth of $z(n)$ when $n$ runs over $\mathcal{E}_{z}^{(m)}$. More precisely,

Theorem 2. Let $A_{m}$ and $\beta_{m}$ be the constants as in Conjecture 1. Then

$$
\sum_{n \in \mathcal{E}_{z}^{(m)}(x)} \frac{z(n)}{n}>\frac{1}{\log \alpha}\left(\frac{\log ^{2} x}{2}-A_{m} \frac{(\log x)^{2-\beta_{m}}}{2-\beta_{m}}+\frac{\beta_{m}}{1-\beta_{m}}(\log x)^{1-\beta_{m}}\right)
$$

as $x$ tends to infinity, where $\alpha=(1+\sqrt{5}) / 2$.

The proof of the theorem combines Diophantine properties of $z(n)$ with analytical tools concerning the average growth rate of multiplicative functions.

\section{Auxiliary Results}

In this section, we shall present some results that will be essential tools in the proof. Throughout this work, we employ the usual Landau "Big $\mathrm{Oh}^{\prime}$ and "Small $\mathrm{Oh}^{\prime}$ notations $\mathrm{O}$ and $o$, respectively, as well as the associated Vinogradov symbols «and $\gg$. We also reserve the letter $p$ for prime numbers.

The first ingredient is a type of "closed formula" for $z(n)$ depending on $z\left(p^{a}\right)$ for all prime factor $p$ of $n$. The proof of this fact may be found in [16].

Lemma 1 (Theorem 3.3 of [16]). Let $n>1$ be an integer with prime factorization $n=p_{1}^{a_{1}} \cdots p_{k}^{a_{k}}$. Then:

$$
z(n)=\operatorname{lcm}\left(z\left(p_{1}^{a_{1}}\right), \ldots, z\left(p_{k}^{a_{k}}\right)\right) .
$$

In general, one has that

$$
z\left(\operatorname{lcm}\left(m_{1}, \ldots, m_{k}\right)\right)=\operatorname{lcm}\left(z\left(m_{1}\right), \ldots, z\left(m_{k}\right)\right) .
$$

For a positive integer $m$, we defined $\mathcal{P}_{m}$ as:

$$
\mathcal{P}_{m}:=\{p: z(p) \equiv 0 \quad(\bmod m)\} .
$$

In 2014, Cubre and Rouse [17] proved that $\# \mathcal{P}_{m}(x)=\left(\rho_{m}+o(1)\right) x / \log x$ for some positive constant $\rho_{m}$, as $x \rightarrow \infty$. Our next tool is a recent result due to Sanna [18] who improved the previous fact by providing the error term of that estimate (furthermore, 
Sanna dealt with the more general context of Lucas sequences). To avoid unnecessary technicalities, we shall state here a consequence of Theorem 1.1 of [18], which is enough for our purposes.

Lemma 2. Let $m$ be a positive integer. Then there exist effectively computable positive constants $a_{m}, b_{m}$ and $c_{m}$, with $\max \left\{a_{m}, b_{m}\right\}<1$, for which

$$
\# \mathcal{P}_{m}(x)=a_{m} \frac{x}{\log x}+O\left(\frac{x}{(\log x)^{1+b_{m}}}\right),
$$

for all $x>c_{m}$

The next three auxiliary results come from the analytic number theory. The first one is the well-known Prime Number Theorem with error estimate.

Lemma 3. Let $\pi(x)$ be the prime counting function. Then:

as $x \rightarrow \infty$.

$$
\pi(x)=\frac{x}{\log x}+O\left(\frac{x}{(\log x)^{2}}\right)
$$

We now recall the theoretical result of Spearman and Williams [19], which is very useful for estimating the order of counting functions (here, as usual, the summatories $\sum_{p \leq x}$ and $\sum_{n \leq x}$ run over the set of prime numbers and positive integers $\leq x$, respectively). Again, we shall state only the sufficient version for this work.

Lemma 4 (Proposition 5.5 of [19]). Let $f: \mathbb{Z}_{\geq 1} \rightarrow \mathbb{R}$ be a multiplicative function with $0 \leq f(n) \leq 1$, for all $n \in \mathbb{Z}_{\geq 1}$. Suppose that there are constants $\tau$ and $\beta$ with $\tau>0$ and $0<\beta<1$ such that

$$
\sum_{p \leq x} f(p)=\tau \frac{x}{\log x}+O\left(\frac{x}{(\log x)^{1+\beta}}\right)
$$

as $x \rightarrow \infty$. Then, there exists a positive computable constant $E$ such that

$$
\sum_{n \leq x} f(n)=E \frac{x}{(\log x)^{1-\tau}}+O\left(\frac{x}{(\log x)^{1+\beta-\tau}}\right)
$$

as $x \rightarrow \infty$.

Our last auxiliary result is a useful formula due to Abel, which makes an interplay between a discrete (summatory) and a continuous (integral) sum.

Lemma 5. (Abel's Summation Formula) Let $\left(a_{n}\right)_{n}$ be a sequence of real numbers and denote its partial sum $A(x):=\sum_{n \leq x} a_{n}$. For a real number $x>1$, let $f$ be a continuously differentiable function on $[1, x]$. Then:

$$
\sum_{n \leq x} a_{n} f(n)=A(x) f(x)-\int_{1}^{x} A(t) f^{\prime}(t) d t .
$$

The proofs of Lemmas 3 and 5 can be found in any good text on analytic number theory (in particular, we refer to [20]).

We are now ready to proceed with the proof of theorems. 


\section{The Proofs}

3.1. The Proof of Theorem 1

We start by defining $\mathcal{F}_{z}^{(m)}$ as

$$
\mathcal{F}_{z}^{(m)}:=\left\{n \in \mathbb{Z}_{\geq 1}: z(n) \not \equiv 0 \quad(\bmod m)\right\} .
$$

Since $[1, x]=\mathcal{E}_{z}^{(m)}(x) \cup \mathcal{F}_{z}^{(m)}(x)$, then, in order to prove (1) it suffices to show that

$$
\# \mathcal{F}_{z}^{(m)}(x) \ll x /(\log x)^{\beta_{m}},
$$

as $x \rightarrow \infty$, for some positive constant $\beta_{m}$. First, we claim that

$$
\mathcal{F}_{z}^{(m)} \subseteq\left\{n \in \mathbb{Z}_{\geq 1}: p \mid n \Rightarrow p \notin \mathcal{P}_{m}\right\}
$$

where $\mathcal{P}_{m}$ is defined right before Lemma 2. Indeed, if $p$ is a prime factor of $n \in \mathcal{F}_{z}^{(m)}$, then (by Lemma 1), $z(p)$ divides $z(n)$. Thus, $m \nmid z(p)$ (otherwise $m$ would divide $z(n)$ contradicting the fact that $n \in \mathcal{F}_{z}^{(m)}$ ) and so $p \notin \mathcal{P}_{m}$. In particular, (3) yields that $\mathcal{F}_{z}^{(m)} \subseteq$ $\mathbb{Z}_{\geq 1} \backslash \mathcal{P}_{m}$ and hence

$$
\# \mathcal{F}_{z}^{(m)}(x) \leq \#\left\{n \leq x: p \mid n \Longrightarrow p \notin \mathcal{P}_{m}\right\},
$$

for all $x \geq 1$.

Now, we need to find an upper bound for the right-hand side of (4). For that, we shall apply Lemma 4 for $f: \mathbb{Z}_{\geq 1} \rightarrow \mathbb{R}$ being the characteristic function of $\mathbb{Z}_{\geq 1} \backslash \mathcal{P}_{m}$, namely,

$$
f(n)= \begin{cases}1, & \text { if } n \in \mathbb{Z}_{\geq 1} \backslash \mathcal{P}_{m} ; \\ 0, & \text { if } n \in \mathcal{P}_{m} .\end{cases}
$$

Since $0 \leq f(n) \leq 1$ and by combining Lemmas 2 and 3 , we deduce the existence of real constants $\beta_{m}, b_{m} \in(0,1)$ such that

$$
\begin{aligned}
\sum_{p \leq x} f(p) & =\#\left\{p \leq x: p \notin \mathcal{P}_{m}\right\} \\
& =\pi(x)-\# \mathcal{P}_{m}(x) \\
& =\frac{x}{\log x}+O\left(\frac{x}{\log ^{2} x}\right)-\left(\beta_{m} \frac{x}{\log x}+O\left(\frac{x}{(\log x)^{1+b_{m}}}\right)\right) \\
& =\tau_{m} \frac{x}{\log x}+O\left(\frac{x}{(\log x)^{1+b_{m}}}\right)
\end{aligned}
$$

as $x \rightarrow \infty$, where $\tau_{m}:=1-\beta_{m}>0$ and we used that $\beta_{m}<1$.

Therefore, the hypotheses of Lemma 4 are fulfilled and so, by that lemma, there exists a positive constant $E_{m}$ such that

$$
\begin{aligned}
\#\left\{n \leq x: n \notin \mathcal{P}_{m}\right\} & =\sum_{n \leq x} f(n) \\
& =E_{m} \frac{x}{(\log x)^{\beta_{m}}}+O\left(\frac{x}{(\log x)^{\beta_{m}+b_{m}}}\right) \\
& \leq A_{m} \frac{x}{(\log x)^{\beta_{m}}},
\end{aligned}
$$


for some constant $A_{m}>0$ and all sufficiently large $x$. Hence, by (4), one has

$$
\begin{aligned}
\mathcal{E}_{z}^{(m)}(x) & \geq x-\# \mathcal{F}_{z}^{(m)}(x) \\
& \geq x-\#\left\{n \leq x: n \notin \mathcal{P}_{m}\right\} \\
& \geq x-A_{m} \frac{x}{(\log x)^{\beta_{m}}},
\end{aligned}
$$

for all sufficiently large $x$, as desired. Note that, by the previous inequality, one has

$$
1 \geq \delta\left(\mathcal{E}_{z}^{(m)}\right)=\lim _{x \rightarrow \infty} \frac{\# \mathcal{E}_{z}^{(m)}(x)}{x} \geq \lim _{x \rightarrow \infty}\left(1-\frac{A_{m}}{(\log x)^{\beta_{m}}}\right)=1
$$

and thus the natural density of $\mathcal{E}_{z}^{(m)}$ is equal to 1 . The proof is then complete.

\subsection{The Proof of Theorem 2}

By definition, one has that $n$ divides $F_{z(n)}$ and so $n \leq F_{z(n)}\left\langle\alpha^{z(n)}\right.$. Thus, $\left.z(n)\right\rangle$ $\log n / \log \alpha$, and therefore:

$$
\sum_{n \in \mathcal{E}_{z}^{(m)}(x)} \frac{z(n)}{n}>\frac{1}{\log \alpha} \cdot \sum_{n \in \mathcal{E}_{z}^{(m)}(x)} \frac{\log n}{n} .
$$

Let $\chi_{m}: \mathbb{Z}_{\geq 1} \rightarrow \mathbb{R}$ be the characteristic function of $\mathcal{E}_{z}^{(m)}$, i.e.,

$$
\chi_{m}(n)= \begin{cases}1, & \text { if } n \in \mathcal{E}_{z}^{(m)} \\ 0, & \text { if } n \notin \mathcal{E}_{z}^{(m)}\end{cases}
$$

Hence,

$$
\frac{1}{\log \alpha} \cdot \sum_{n \in \mathcal{E}_{z}^{(m)}(x)} \frac{\log n}{n}=\frac{1}{\log \alpha} \cdot \sum_{n \leq x} \chi_{m}(n) \frac{\log n}{n} .
$$

Now, by taking $a_{n}:=\chi_{m}(n)$ and $f(x):=(\log x) / x$ in Lemma 5 , we have that $A(x)=\# \mathcal{E}_{z}^{(m)}(x), f^{\prime}(x)=(1-\log x) / x^{2}$ and so

$$
\sum_{n \leq x} \chi_{m}(n) \frac{\log n}{n}=\# \mathcal{E}_{z}^{(m)}(x) \frac{\log x}{x}+\int_{1}^{x}\left(\frac{\log t-1}{t^{2}}\right) \# \mathcal{E}_{z}^{(m)}(t) d t .
$$

By using Theorem 1 for the first term of the right-hand side of (6), one obtains:

$$
\begin{aligned}
\# \mathcal{E}_{z}^{(m)}(x) \frac{\log x}{x} & \geq\left(x-A_{m} \frac{x}{(\log x)^{\beta_{m}}}\right) \cdot \frac{\log x}{x} \\
& =\log x-A_{m}(\log x)^{1-\beta_{m}}
\end{aligned}
$$

as $x \rightarrow \infty$.

Again, Theorem 1 applied to the integrand of the second term of the right-hand side of (6) yields:

$$
\begin{aligned}
\left(\frac{\log t-1}{t^{2}}\right) \# \mathcal{E}_{z}^{(m)}(t) & \geq\left(\frac{\log t-1}{t^{2}}\right)\left(t-A_{m} \frac{t}{(\log t)^{\beta_{m}}}\right) \\
& =\frac{\log t}{t}-A_{m} \frac{(\log t)^{1-\beta_{m}}}{t}-\frac{1}{t}+A_{m} \frac{1}{t(\log t)^{\beta_{m}}}
\end{aligned}
$$


for all $x$ large enough. By integrating the previous inequality and after a straightforward calculation, we obtain that

$$
\int_{1}^{x}\left(\frac{\log t-1}{t^{2}}\right) \# \mathcal{E}_{z}^{(m)}(t) d t \geq \frac{\log ^{2} x}{2}-A_{m} \frac{(\log x)^{2-\beta_{m}}}{2-\beta_{m}}-\log x+A_{m} \frac{(\log x)^{1-\beta_{m}}}{1-\beta_{m}},
$$

where we evaluate the (convergent) improper integral $\int_{1}^{x} \frac{1}{t(\log t)^{\beta m}} d t$ by

$$
\begin{aligned}
\int_{1}^{x} \frac{1}{t(\log t)^{\beta_{m}}} d t= & \lim _{\epsilon \rightarrow 1^{+}} \int_{\epsilon}^{x} \frac{1}{t(\log t)^{\beta_{m}}} d t=\frac{1}{1-\beta_{m}} \lim _{\epsilon \rightarrow 1^{+}}\left((\log x)^{1-\beta_{m}}-(\log \epsilon)^{1-\beta_{m}}\right)=\frac{(\log x)^{1-\beta_{m}}}{1-\beta_{m}}, \\
& \text { since }(\log \epsilon)^{1-\beta_{m}} \text { tends to } 0 \text { as } \epsilon \rightarrow 1^{+}\left(\text {because } 1-\beta_{m}>0\right) .
\end{aligned}
$$

Now, by combining (5), (7) and (8), we arrive at

$$
\sum_{n \in \mathcal{E}_{z}^{(m)}(x)} \frac{z(n)}{n}>\frac{1}{\log \alpha}\left(\frac{\log ^{2} x}{2}-A_{m} \frac{(\log x)^{2-\beta_{m}}}{2-\beta_{m}}+\frac{\beta_{m}}{1-\beta_{m}}(\log x)^{1-\beta_{m}}\right)
$$

as $x \rightarrow \infty$, which finishes the proof.

\section{Conclusions}

In this paper, we proved a conjecture related to the order of appearance in the Fibonacci sequence function $z: \mathbb{Z}_{\geq 1} \rightarrow \mathbb{Z}_{\geq 1}$, defined as $z(n):=\min \left\{k \geq 1: n \mid F_{k}\right\}$. Recently, Trojovská and Venkatachalam [14] showed that the natural density of $\{n \geq 1: z(n) \equiv 0$ $\left.\left(\bmod 2^{k}\right)\right\}$ is equal to 1 , for any $k \geq 1$ previously fixed. Furthermore, they conjectured that the same holds for the set of positive integers $n$ for which $m \mid z(n)$ (for any given integer $m \geq 1$ ). In this work, we confirmed this conjecture: for any $m \geq 1$, the natural density of the set $\{n \geq 1: z(n) \equiv 0(\bmod m)\}$ is equal to 1 . Moreover, we provided a lower bound for $\sum_{n \leq x} z(n) / n$, where $n$ runs over the set $\{n \geq 1: m \mid z(n)\}$. The proofs combine arithmetical and analytic tools in number theory.

There are some natural future developments of this work, as, for example, the study of the order of appearance in related number sequences, such as generalized Fibonacci numbers and Pell-Lucas numbers might lead to interesting results as well.

Author Contributions: Formal analysis, E.T.; Funding acquisition, E.T.; Methodology, E.T.; Software, V.K.; Supervision, E.T.; Validation, V.K.; Writing (original draft), V.K. All authors have read and agreed to the published version of the manuscript.

Funding: The research was supported by the Excellence Project PřF UHK No. 2213/2021-2022., University of Hradec Králové, Czech Republic.

Institutional Review Board Statement: Not applicable.

Informed Consent Statement: Not applicable.

Acknowledgments: The authors thank the University of Hradec Králové for its support.

Conflicts of Interest: The authors declare no conflict of interest.

\section{References}

1. Lucas, E. Théorie des fonctions numériques simplement périodiques. Am. J. Math. 1878, 1, 289-321. [CrossRef]

2. Sallé, H.J.A. Maximum value for the rank of apparition of integers in recursive sequences. Fibonacci Quart. 1975, 13, 159-161.

3. Sloane, N.J.A. The On-Line Encyclopedia of Integer Sequences, Published Electronically. Available online: http://www.oeis.org/ (accessed on 20 October 2021).

4. Trojovský, P. Some problems related to the growth of $z(n)$. Adv. Differ. Equ. 2020, 2020, 270. [CrossRef]

5. Marques, D. Sharper upper bounds for the order of appearance in the Fibonacci sequence. Fibonacci Quart. 2013, 51, $233-238$.

6. Marques, D. Fixed points of the order of appearance in the Fibonacci sequence. Fibonacci Quart. 2012, 50, 346-352.

7. Somer, L.; Kř́žžek, M. Fixed points and upper bounds for the rank of appearance in Lucas sequences. Fibonacci Quart. 2013, 51, 291-306.

8. Sun, Z.H.; Sun, Z.W. Fibonacci numbers and Fermat's last theorem. Acta Arith. 1992, 60, 371-388. [CrossRef] 
9. Luca, F.; Tron, E. The distribution of self-Fibonacci divisors, Advances in the theory of numbers. In Fields Institute Communications; Fields Institute Research Mathematical Science: Toronto, ON, Canada, 2015; Volume 77, pp. 149-158.

10. Trojovský, P. On Diophantine equations related to the order of appearance in Fibonacci sequence. Mathematics 2019, 7, 1073. [CrossRef]

11. Trojovská, E. On the Diophantine Equation $z(n)=(2-1 / k) n$ Involving the Order of Appearance in the Fibonacci Sequence. Mathematics 2020, 8, 124. [CrossRef]

12. Trojovský, P. On the Natural Density of Sets Related to Generalized Fibonacci Numbers of Order $r$. Axioms 2021, 10, 144. [CrossRef]

13. Trojovský, P. On the Parity of the Order of Appearance in the Fibonacci Sequence. Mathematics 2021, 9, 1928. [CrossRef]

14. Trojovská, E. Venkatachalam, K. The Proof of a Conjecture Related to Divisibility Properties of $z(n)$. Mathematics 2021, 9, 2638. [CrossRef]

15. Trojovský, P. On the Growth of Some Functions Related to $z(n)$. Mathematics 2020, 8, 876. [CrossRef]

16. Renault, M. Properties of the Fibonacci Sequence Under Various Moduli. Master's Thesis, Wake Forest University, Winston-Salem, NC, USA, 1996. Available online: http:/ / webspace.ship.edu/msrenault/ fibonacci/FibThesis.pdf (accessed on 20 October 2021).

17. Cubre, P.; Rouse, J. Divisibility properties of the Fibonacci entry point. Proc. Am. Math. Soc. 2014, 142, 3771-3785. [CrossRef]

18. Sanna, C. On the Divisibility of the Rank of Appearance of a Lucas Sequence. Available online: https://arxiv.org/abs/2008.12506 (accessed on 20 October 2021).

19. Spearman, B.K.; Williams, K.S. Values of the Euler phi function not divisible by a given odd prime. Ark. Mat. 2006, 44, 166-181. [CrossRef]

20. De Koninck, J.-M.; Luca, F. Analytic Number Theory-Exploring the Anatomy of Integers. In Analytic Number Theory: Exploring the Anatomy of Integers; American Mathematical Soc.: Providence, RI, USA, 2012. 